\title{
Expiratory CT: What is Good Enough?
}

\section{Mary Salvatore, MD, MBA ${ }^{1,2^{*}}$, Lea Azour, $M D^{1,3}$, Mary $O^{\prime} C_{0 n n o r}{ }^{1}, K M$ Capaccione, MD, PhD and David Mendelson, MD $^{1}$}

${ }^{1}$ Department of Radiology, Icahn School of Medicine at Mount Sinai, USA

${ }^{2}$ Department of Radiology, Columbia University Medical Center, USA

${ }^{3}$ Department of Radiology, NYU Langone Health, USA

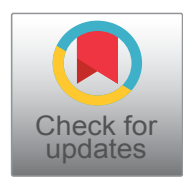

*Corresponding author: Mary Salvatore, MD, MBA, Department of Radiology, Icahn School of Medicine at Mount Sinai, USA; Department of Radiology, Columbia University Medical Center, New York 10029, USA

\begin{abstract}
Purpose: To determine the difference in craniocaudal dimension of an inspiratory CT scan minus an expiratory CT scan that constitutes an average expiratory effort to potentially exclude air trapping.

Methods: This study obtained approval from the Institutional Review Board. The craniocaudal dimensions of inspiratory and expiratory chest CT scans of 83 patients were examined by an experienced radiologist (MS with 20 years of experience). The difference between the two measurements, called the Slice Level Difference (SLD), was calculated. Mosaic attenuation on inspiratory images and air trapping on expiratory images were quantified, as well as the change in grade between the two series. Age and gender were compared to degree of expiratory effort.
\end{abstract}

Results: Of the 83 patients in our study, air trapping was found on 49 of the expiratory images. Patients with air trapping on expiratory images had an average SLD of $32.1 \mathrm{com}$ pared to those without air trapping who had an average SLD of 25.7. Furthermore, $63 \%$ of those with air trapping had a SLD greater than or equal to 30 , whereas only $41 \%$ with lower SLD had air-trapping. The average age was younger for those with a SLD $\geq 30$ (56 years versus 63 years). In addition, a greater percentage of males had a larger SLD.

Conclusion: Adequate expiratory CT imaging allows recognition of air trapping when present. Not all expiratory CT scans are performed well enough to diagnose air trapping. Comparing SLD between inspiratory and expiratory images can help determine if there was greater than average expiratory effort to potentially exclude the diagnosis of small airways disease.

\section{Introduction}

Inspiratory chest CT scan is the standard method for viewing the lung parenchyma. Inspiratory images are insufficient for the diagnosis of air trapping. Often there is no evidence of mosaic attenuation on inspiratory CT scans when subsequent expiratory images demonstrate air trapping [1-3]. Limited sensitivity for mosaic attenuation on inspiratory CT scans supports the use of expiratory imaging when air trapping is suspected [4-7].

A pitfall of expiratory imaging is its dependence on patient effort, and reproducibility. Patients must blow out as much air as possible and refrain from inhaling for approximately ten seconds, which can be difficult for those with decreased lung function. Obstacles in obtaining images based on optimal expiratory effort may occur when the patient is unable to hear or understand breathing instructions. Inability or unwillingness to follow directions can result in non-diagnostic images, with increased radiation dose and no added benefit.

The primary goal of this study is to evaluate a metric to determine patient effort on expiratory CT scan. The secondary goal is to examine factors associated with poor expiratory exams.

\section{Materials and Methods}

This HIPAA compliant, IRB approved research project studied 83 consecutive subjects who received both inspiratory and expiratory chest CT scans for a variety of indications at Mount Sinai Hospital during the year 2016. The chest CT scans were performed using standard low dose technique. Age, sex and reason for CT scan were documented. All CT scans, both inspiratory 
and expiratory, were reviewed by a single radiologist (MS with 20 years' experience). The slice level, which is noted on each CT image, of the apex and the base of the right lung was recorded during inspiration and expiration for each subject. The apex was defined as the first image where lung was identified. The base was defined as the first image where diaphragm was identified. Next, the slice level difference (SLD) between apex and base of the expiratory CT scan was subtracted from the slice level difference of the inspiratory $\mathrm{CT}$ scan yielding the total slice level difference (SLD). A lower number indicated little difference in the cranio-caudal dimension of the lung between inspiratory and expiratory images and was considered a poor expiratory effort. Likewise, a large total SLD was consistent with a better effort.

All images were inspected by the radiologist for signs of mosaic attenuation [8] and air trapping, which were graded on the inspiratory and expiratory scans of each subject, respectively. A grade of 0 was assigned to a scan where $0 \%$ of the lungs were impacted by mosaic attenuation or air-trapping, a grade of 1 was assigned to a scan where 1 lobe of the lung was impacted by mosaic attenuation or air-trapping, a grade of 2 was assigned to a scan where 2 lobes of the lung were impacted by mosaic attenuation or air-trapping, a grade of 3 was assigned to a scan where 3 lobes of the lungs were impact-

Table 1: Summative data

\begin{tabular}{|l|l|}
\hline \# of Patients & 83 \\
\hline \% male & $52 / 83(63 \%)$ \\
\hline Average age & 59 \\
\hline Percent with normal initial CT & $69 / 83(83 \%)$ \\
\hline Percent with change is mosaic attenuation & $49 / 83(59 \%)$ \\
\hline Average slice level difference & 31.3 \\
\hline $\begin{array}{l}\text { \% with Slice level difference greater than or } \\
\text { equal 30 }\end{array}$ & $45 / 83(54 \%)$ \\
\hline
\end{tabular}

ed by mosaic attenuation or air-trapping, and a grade of 4 was assigned to a scan where $\geq 4$ lobes of the lungs were impacted by mosaic attenuation or air-trapping. These grades were compared to SLD.

\section{Results}

83 consecutive patients who had inspiratory and expiratory CT scans of the chest on the same day were included in the study. $63 \%$ were males. The average age was 59 -years-old. $69 / 83$ had no evidence of mosaic attenuation (heterogeneity of the lung parenchyma) on initial exam. On expiratory CT scans, $49 / 83$ had a change in extent of heterogeneity of lung parenchyma, which is the definition of air trapping [9]. The average SLD for all patients was 31.3 (Table 1). 54\% had a SLD $\geq 30$.

Table 2 compares SLD $<30$ to SLD $\geq 30$. Patients with poor effort $(<30$ SLD) were older and less likely to demonstrate air trapping than those with good effort ( $\geq 30$ SLD). A larger percentage of men had a SLD greater than or equal to 30 . There was a larger percent of change in mosaic attenuation for patients with SLD $\geq$ 30 ( $47 \%$ vs. $69 \%)$.

Table 3 compares patients with air trapping to those without air trapping which is defined as increased mosaic attenuation on expiratory images. The average age for both groups is 59. There are a larger percentage of men in the group without air-trapping. There is a larger average SLD and higher percentage of patients with $S L D \geq 30$ in group with air trapping. The smallest SLD to demonstrate a change in extent of mosaic attenuation from inspiration to expiration was five but the average was SLD was 32.

\section{Discussion}

Mosaic attenuation on inspiratory chest $\mathrm{CT}$ is a nonspecific diagnosis, which means that the parenchyma is

Table 2: Comparing those with slice level distance less than or greater than or equal to 30 .

\begin{tabular}{|l|l|l|}
\hline & $<\mathbf{3 0}$ SLD Poor effort & $\geq 30$ SLD Good effort \\
\hline \# of Patients & 38 & 45 \\
\hline \% male & $21 / 38(55 \%)$ & $31 / 45(69 \%)$ \\
\hline Average age & 63 & 56 \\
\hline Percent normal on initial CT & $29 / 38(76 \%)$ & $40 / 45(89 \%)$ \\
\hline Average slice level difference & 15 & 41 \\
\hline Percent with change in mosaic attenuation & $18 / 38(47 \%)$ & $31 / 45(69 \%)$ \\
\hline
\end{tabular}

Table 3: Comparing those with air trapping verses those without on expiratory images.

\begin{tabular}{|l|l|l|}
\hline & Patients with Air Trapping & Patients without Air Trapping \\
\hline \# of Patients & 49 & 34 \\
\hline$\%$ male & $57 \%$ & $71 \%$ \\
\hline Average age & 59 & 59 \\
\hline Percent with normal initial CT & $42 / 49(86 \%)$ & $27 / 34(79 \%)$ \\
\hline Average slice level difference & 32.1 & 25.7 \\
\hline \% with Slice level difference greater than or equal 30 & $31 / 49(63 \%)$ & $14 / 34(41 \%)$ \\
\hline
\end{tabular}


heterogeneous. Heterogeneous parenchyma can occur with diseases of the alveoli, pulmonary artery or small airways. When mosaic attenuation becomes more pronounced on expiration the patient is said to have air tapping. Secondary findings of bronchial wall thickening support the diagnosis.

Air trapping on CT scans can be seen in normal patients and those with asthma, bronchiolitis obliterans and chronic hypersensitivity pneumonitis, among others. The presence of air trapping changes the differential diagnosis. Radiologists have typically looked at the posterior tracheal wall to determine if a CT scan is in the expiratory phase. This may be difficult given variable reports of tracheal morphology with expiration, including cases in which a patient's posterior tracheal wall does not demonstrate significant change upon expiration. In addition, some trachea have atypical shapes on inspiration making the comparison challenging. Measuring the cranial caudal dimension evaluates the location of the diaphragm, a major contributor to expiratory effort. The total SLD is an ideal measurement as it corrects for slice thickness.

To our knowledge, there is no reported number for SLD to signify adequate expiratory effort. The average measurement of SLD in our patient population was 31, which may serve as a rough benchmark. Similarly, a study of diaphragmatic motion by ultrasonography noted diaphragmatic excursion of $3.7 \mathrm{~cm}$ for women and $4.7 \mathrm{~cm}$ for men during deep breathing [10], corroborating our findings of increased average SLD in men. Assessing the adequacy of expiratory imaging is important because air trapping on expiratory images supports a diagnosis of small airways disease and lack of a diagnosis of small airway disease will prompt the clinician to investigate other causes for dyspnea.

Patients may fail to achieve adequate expiratory effort for a variety of reasons including language barrier, lack of understanding, or physical limitation. To overcome these obstacles, attention to patients' ability to comply with breathing instructions should be considered, and clinicians and technologists may consider coaching patients with breathing instructions prior to scanning. We found that in general younger male patients had a better expiratory effort as judged by a larger SLD than older female patients, suggesting multiple potential confounding factors.

Our study had several limitations. We did not correlated SLD with patient factors that may be influential and require further investigation, such as body surface area, body mass index concomitant emphysema or chronic obstructive pulmonary disease. The influence of diaphragmatic eventration was not assessed. Future study may benefit from correlation with spirometry. We did not study dynamic expiratory CT scans, potentially beneficial, and a topic of further investigation.
In conclusion, SLD is an easily applicable technique that may be used to determine if expiratory chest CT images are satisfactory. Expiratory CT scans are necessary for the diagnosis of air trapping, as mosaic attenuation may not be identified on inspiratory CT scans in up to $50 \%$ of patients studied, who ultimately had air trapping on expiratory images. Identification of air trapping on expiratory CT scans narrows differential considerations for dyspnea, and conversely, reliable exclusion of air trapping is necessary and often dependent on adequate expiratory imaging.

\section{Disclosures}

Mary Salvatore, MD, MBA- Speaker and Consultant: Genentech, Boehringer Ingelheim. Grant funding Genentech, Boehringer Ingelheim.

\section{References}

1. Arakawa H, Webb WR (1998) Air trapping on expiratory high-resolution ct scans in the absence of inspiratory scan abnormalities: correlation with pulmonary function tests and differential diagnosis. AJR Am J Roentgenol 170: 13491353.

2. Gaeta M, Minutoli F, Girbino G, Murabito A, Benedetto C, et al. (2013) Expiratory CT scan in patients with normal inspiratory CT scan: A finding of obliterative bronchiolitis and other causes of bronchiolar obstruction. Multidisciplinary Respiratory Medicine 8.

3. Arakawa H, Webb WR, McCowin M, Katsou G, Lee KN, et al. (1998) Inhomogeneous lung attenuation at thin-section CT: Diagnostic value of expiratory scans. Radiology 206: 89-94.

4. Zhang J, Hasegawa I, Hatabu H, Feller-Kopman D, Boiselle PM (2004) Frequency and severity of air trapping at dynamic expiratory CT in patients with tracheobronchomalacia. AJR Am J Roentgenol 182: 81-85.

5. Arakawa $H$, Niimi $H$, Kurihara $Y$, Nakajima $Y$, Webb WR (2000) Expiratory high-resolution CT: Diagnostic value in diffuse lung diseases. AJR Am J Roentgenol 175: 15371543.

6. Miller WT, Chatzkel J, Hewitt MG (2014) Expiratory air trapping on thoracic computed tomography a diagnostic subclassification. Ann Am Thorac Soc 11: 874-881.

7. Stern EJ, Frank MS (1994) Small-airway diseases of the lungs: Findings at expiratory CT. AJR Am J Roentgenol 163: $37-41$

8. Ridge CA, Bankier AA, Eisenberg RL (2011) Mosaic attenuation. AJR 197: W970-W977.

9. Webb WR (1994) High-resolution computed tomography of obstructive lung disease. Radiol Clin North Am 32: 745757.

10. Boussuges A, Gole Y, Blanc P (2009) Diaphragmatic motion studied by M-mode ultrasonography: Methods, reproducibility, and normal values. Chest 135: 391-400.

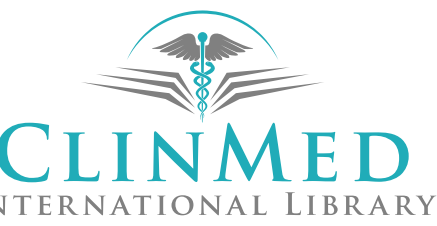

